\title{
SIMULATION OF ROBOT FOR USE IN RESCUE OPERATION GROUPS
}

\author{
Ivan Vasilyev, Alla Kashourina, Maxim Krasheninnikov \& Ekatherina Smirnova
}
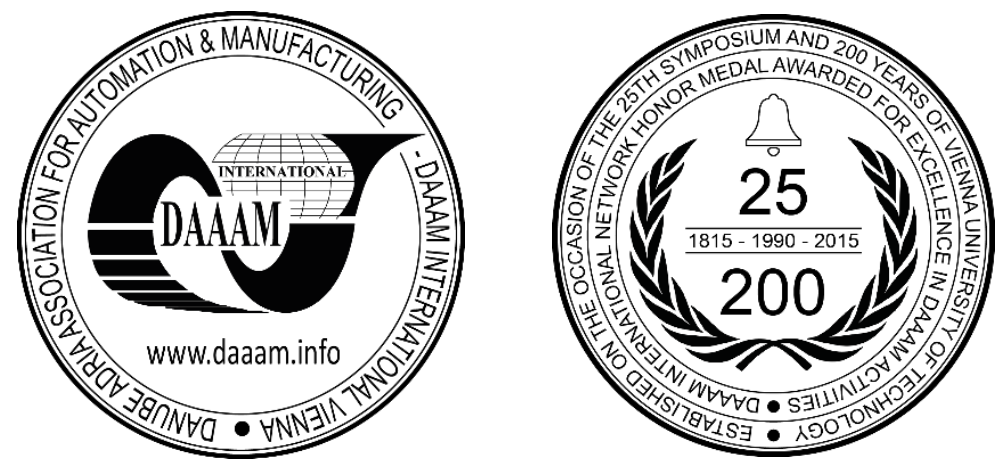

This Publication has to be referred as: Vasilyev, I[van]; Kashourina, A[1la]; Krasheninnikov, M[axim] \& Smirnova, E[katerina] (2016). Simulation of Robot for Use in Rescue Operation Groups, Proceedings of the 27th DAAAM International Symposium, pp.0435-0440, B. Katalinic (Ed.), Published by DAAAM International, ISBN 978-3-90273408-2, ISSN 1726-9679, Vienna, Austria

DOI: $10.2507 / 27$ th.daaam.proceedings.064

\section{Abstract}

The rescue of people in disaster through autonomous means of evacuation in some cases is the only way to save their lives. Rescue Mission often takes place in remote locations. The paper studies modeling of a universal rescue vehicle (URV) with a rotary-screw propeller. URV s provided with a pivotally fastened screws. This method of attachment allows to overcome significant obstacles. There is a problem of management and simulation of such screws. This article describes mathematically the kinematic model of the URV.

Keywords: robot; rescue mission; vehicle.

\section{Introduction}

Universal rescue vehicle (URV) is a passenger all-terrain vehicle of amphibious type, which is provided with four screw propellers, two on each side of the vehicle (Fig. 1).

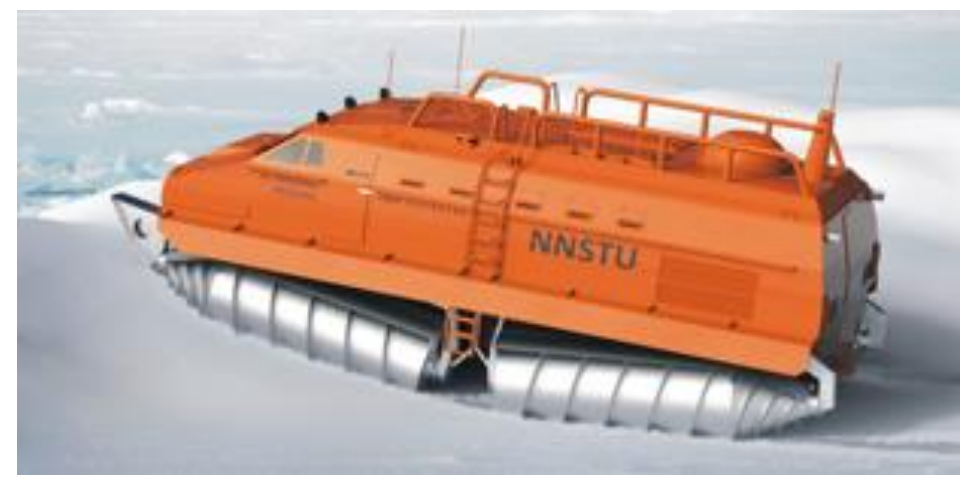

Fig. 1. URV 
Figure 1 illustrates the reason for using "breaking" screws, which is the increase of cross-country motion. Another reason for the use of screw propellers is connected with providing floatation on the water, because screws are hollow and their volume provides additional positive floatability of the whole vehicle. Each screw has an additional actuator for changing its tilt.

Thus, a universal rescue vehicle can move both on hard road and non-cohesive supporting base, including water floatation. And also to overcome the obstacles of considerable height (up to $2 \mathrm{~m}$ ). Let's consider the kinematic scheme of the Universal rescue vehicle (Fig. 2).

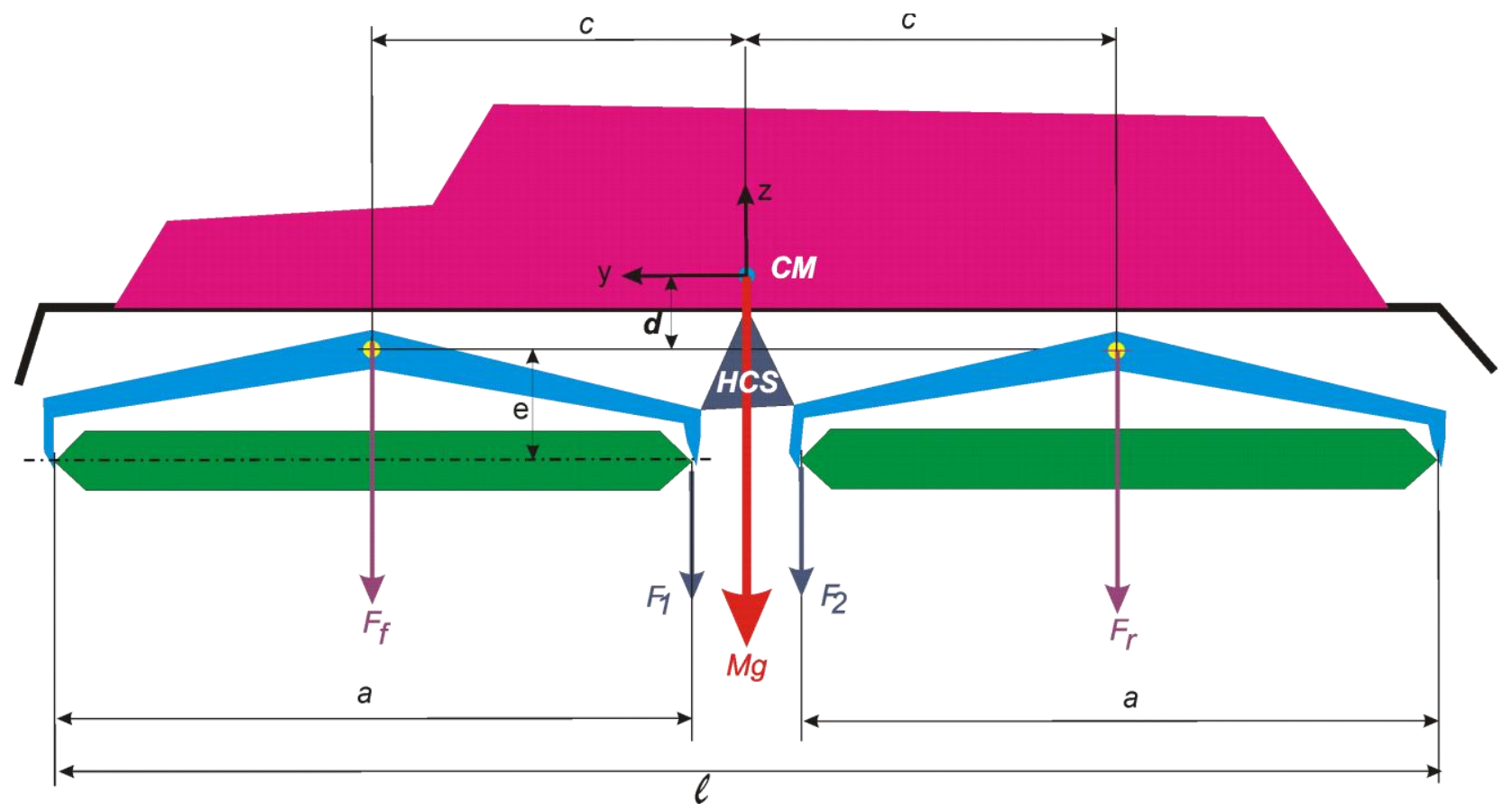

Fig. 2 The kinematic scheme of the URV

In this scheme we denote: CM - center of mass. The fact that CM is exactly halfway between the axes of the mounting screws is quite logical - for the same load of the screws when moving horizontally. In a real situation, of course, it can be (and it is) not. There are many possible reasons for it: from different amount of residual fuel in the tanks to improper seating of passengers in the compartment. But here we consider the situation when everything is perfect. Then we denote: $\mathrm{Mg}$ - gravity of the USV center of mass, axes y and $\mathrm{z}$ denote the base coordinate system (BCS) of the unit (it's more logical to place it in the center of mass), FП - is a component of the USV force of gravity, attributable to the front screw, F3 - a component of the URV gravity attributable to the rear screw.

For simplicity, we assume that the URV is provided with only two screws - front and rear. We "forget" that the URV has a third dimension - width and, accordingly, four screws. For an ideal situation, which is shown in Figure 2, front and rear powers will be equal and will be exactly half of the USV gravity. Abbreviation SHC denotes the system of hydraulic cylinders, providing rotation of the screws. Accordingly, the strengths of these cylinders are designated as F1 and F2. In the described ideal situation, these two forces are equal to zero.

\section{Model of the cylinder in water}

We assume the distance from the BSC to the axes of rotation of the screws in a horizontal plane is denoted as $c$ and a vertical shift as $d$.

Let's try to calculate the depth of immersion of a hollow cylinder of known weight and dimensions. For simplicity, we assume that we just have a cylinder without cone "caps" at the ends. Let the ratio of the weight of the cylinder and the weight of the displaced water be denoted by $\xi$.

The first case is the cylinder stands in water vertically. This option does not seem the most obvious, but it is the easiest, so we will consider it at first. Let's consider Figure 3, which shows the cylinder in water. 


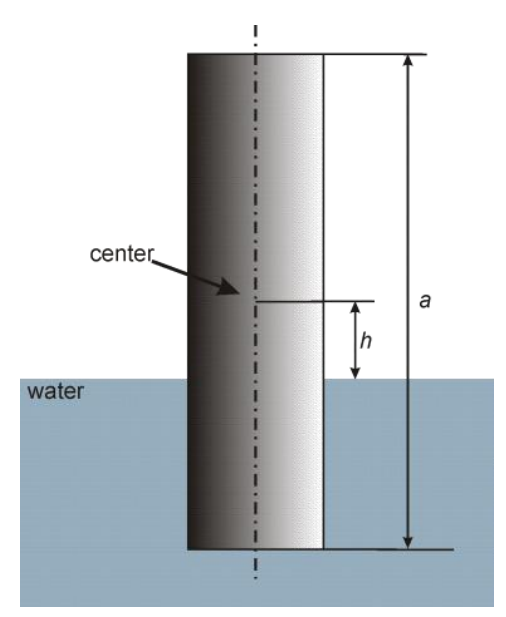

Fig. 3. The cylinder stands vertically in water

Here, the required distance $h$ is easy to find:

$$
h=a\left(\frac{1}{2}-\xi\right)
$$

here all symbols are denoted above.

The second case - the cylinder lies horizontally in water. Here the situation is more complicated, let's consider Figure 4.

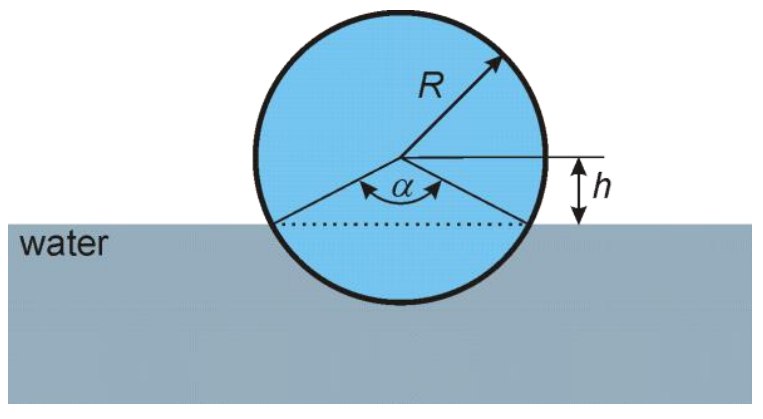

Fig. 4. The cylinder lies horizontally in water. The end view.

Obviously, the ratios of volumes $\xi$ are equal to the ratio of areas of a circle segment below the dotted line and the area of the circle. Therefore we can three-dimensional problem bring to a flat one, shown in Figure 4.

It is easy to obtain a formula for the ratio of the volumes:

$$
\frac{\operatorname{Arccos} \frac{h}{R}}{2 \pi}-\frac{h}{\pi R^{2}} \sqrt{R^{2}-h^{2}}=\xi
$$

This shows that the equation for the desired height $h$ is strongly nonlinear, which is bad. We substitute $\operatorname{Arccos} \frac{h}{R}$ with $\frac{\pi}{2}-1.25 \frac{h}{R}$ that is justified because graphics for modeling coincide - Figure 5

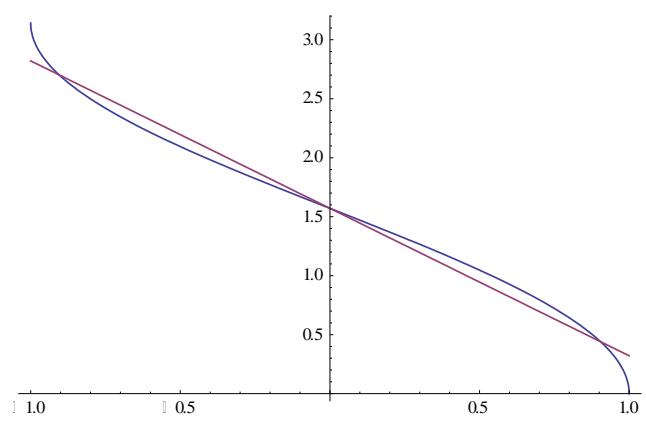

Fig. 5. Graphics Arccos x (blue curve) and $\pi / 2-1.25 x$ (red curve) 
In the result we get equation of the 4th degree, the solution of which is expressed by a "long" formula, which occupies an entire page. No need to bring it here.

Again, for modeling purposes it is sufficient to substitute the circle by the square of the same area - see Fig. 6.

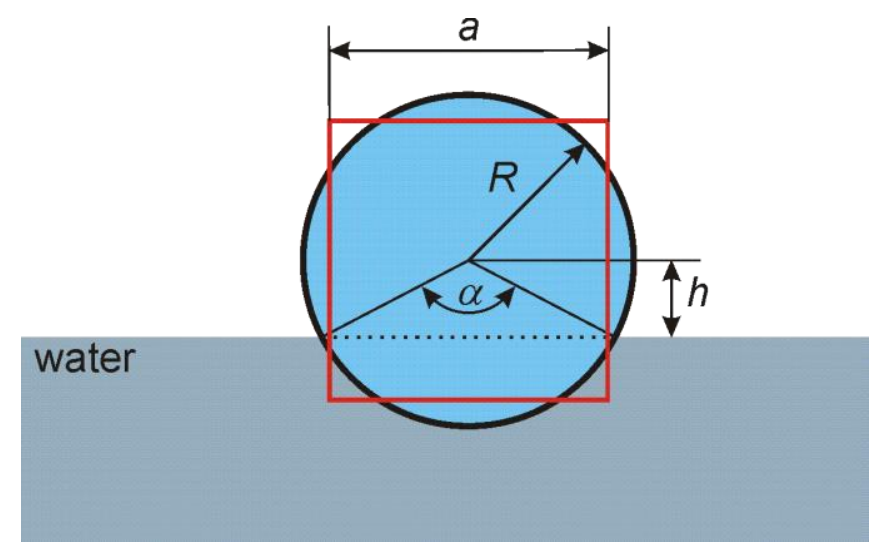

Fig. 6. Replacement of the circle with the square

Here $a$ - is the size of the side of the square. In order to have the same area with the circle the square must have sides equal to:

$a=\sqrt{\pi} R$

Now, we get the familiar expression for the height $h$ :

$h=a\left(\frac{1}{2}-\xi\right)$

Third, the most common case - when the cylinder is at an angle $\alpha$ to the water surface - see Fig. 7.

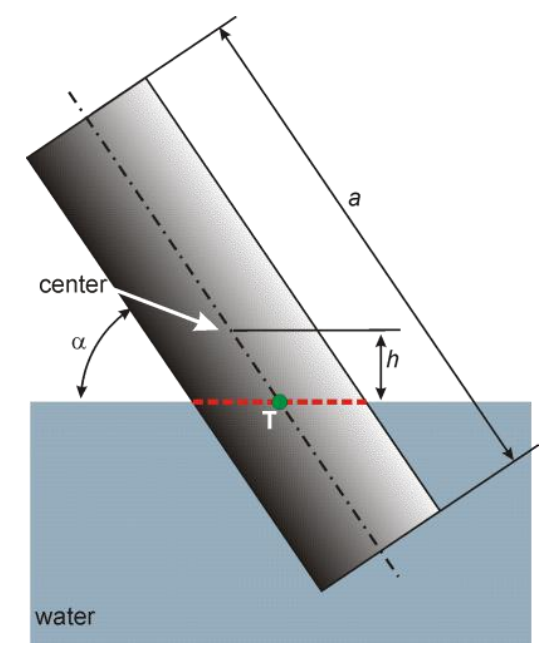

Fig. 7. The cylinder is tilted

Here, the red dotted line shows the level of the water surface. When tilting the cylinder it can be assumed that the cylinder is rotated around the point T, highlighted in Figure 7 as a green dot. Therefore, the value of $h$ is calculated by a simple formula:

$h=a\left(\xi-\frac{1}{2}\right) \cos \alpha$.

Modeling of driving of the universal rescue vehicle from the water to the ice. Based on these formulas, we can calculate the position of the URV at its various positions, both in water and when driving over hard surfaces (shore or ice). Let's consider the efforts that arise in such movements - see Fig. 8. 


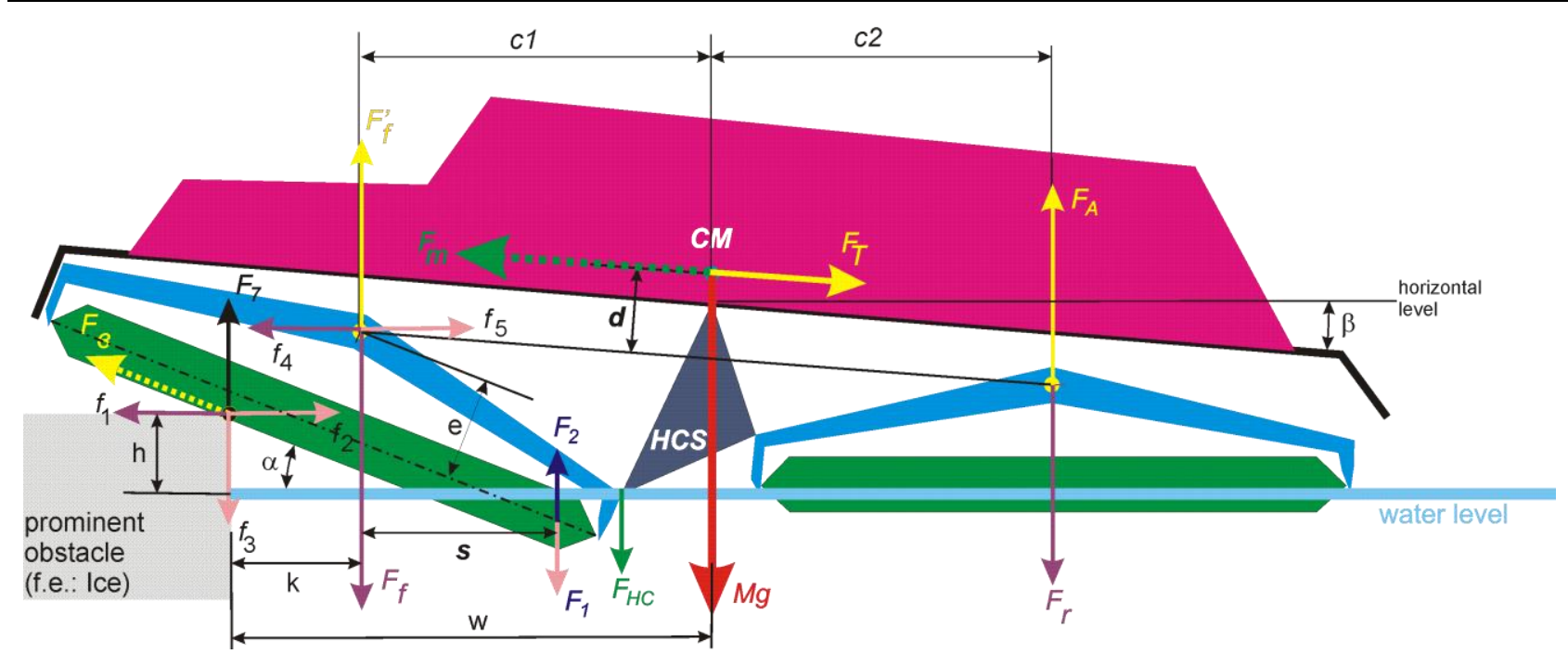

Fig. 8. Demonstration of driving on an obstacle

The following forces are denoted here:

- $\mathrm{Mg}$ - weight of the URV;

- $\mathrm{F}_{\mathrm{A}}$ и $\mathrm{F}_{\text {п }}^{\prime}$ - reaction in the suspension point of the screws;

- $\mathrm{F}_{3}$ и $\mathrm{F}_{\text {п }}$ - load at the point of suspension of the screws;

- $\mathrm{F}_{\text {гц }}$ - the force of the hydraulic cylinder by the tilted screw;

- $\mathrm{F}_{1}$ и $\mathrm{F}_{2}$ - part of the gravity attributable to the front end of the screw and the corresponding buoyancy force;

- $\mathrm{F}_{7}, f_{1}, f_{2}$ и $f_{3}$-forces arising from the support of the front screw on the obstacle;

- $\mathrm{F}_{\mathrm{B}}$ - the strength of the screw screwed into an obstacle;

- $\mathrm{F}_{\mathrm{m}}$ - tractive force required for the movement of the unit.

To raise an obstacle the screwing force $\mathrm{F}_{\mathrm{B}}$ is not enough because this force depends on the pressure force $f_{3}$, and it is may be inadequate at the initial moment because it depends on the values of $w$ и $F_{1}$ :

$f_{3}=M g \frac{c_{2}}{w}-F_{1}$

Traction force of screw in water is negligible, so the URV must be equipped with additional propulsion - a water jet.

Knowing the linking coefficient of the prominent obstacle, which is similar to the coefficient of friction, it is possible to calculate the force required for the movement of the Universal rescue vehicle:

$F_{m}=F_{\mathrm{B}} \frac{\cos \alpha}{\cos \beta}+F_{T}$

where

$\alpha$ - the angle of the screw to the horizon;

$\beta$ - the angle of URV body to the horizon угол;

$F_{T}$ - the water jet traction force.

Let's calculate the required tractive force of the water jet. When moving the unit the main force of resistance is the force of gravity, as the URV is being lifted onto the obstacle.

Therefore, the force $f_{3}$ to be projected onto the axis of the force $\mathrm{F}_{\mathrm{B}}$

$f_{3} \sin \alpha=F_{T} \frac{\cos \beta}{\cos \alpha}+F_{\mathrm{B}}=F_{T} \frac{\cos \beta}{\cos \alpha}+\vartheta f_{3} \cos \alpha$

Where $\vartheta$ - the linking coefficient of the screw on the material obstacles. So the required water jet tractive force:

$F_{T}=\frac{\cos \alpha}{\cos \beta} f_{3}(\sin \alpha-\vartheta \cos \alpha)$ 


\section{Conclusion}

In the article the problem of management and simulation URV. It demonstrated mathematically the kinematic model of URV.

Work on modeling and the creation of a universal rescue vehicle is only at the very beginning. Nevertheless, it is clear that the crossing possibility of the URV in almost any conditions is almost limitless. At present the prototype of the vehicle is being manufactured it will begin to be tested this year.

\section{References}

[1] Briskin E.S, Chernyshev V.V, Maloletov A.V., Sharonov N.G. Comparative analysis of wheeled, tracked and walking machines. // Scientific and Technical Journal «Robotics and Technical Cybernetics», №1, 2013

[2] Screw Rotary rover. Electronic resource: https://en.wikipedia.org/wiki/Screw-propelled_vehicle

[3] Naumov V.N., Mashkov K.Y., Byakov K.E., Modeling of linear motion of transport and technological unit with rotary-screw propeller. // Journal «Engineering», №12-2013

[4] Naumov V.N., Byakov K.E., Change of towing speed characteristics of transport and technological unit with rotaryscrew propulsion by varying the lifting angle of helical blade in the process of motion. // Vestnik MSTU. NE Bauman: electronic edition. 2013

[5] Vasilyev, A. Kashourina, M. Krasheninnikov, E. Smirnova. Use mobile robots groups for rescue missions in extreme climatic conditions // Annals of 25th DAAAM International Symposium on Intelligent Manufacturing and Automation, DAAAM 2014

[6] Smirnova, E.; Stepanov, D. \& Goryunov, V.: A Technique of Natural Visual Landmarks Detection and Description for Mobile Robot Cognitive Navigation // Annals of 26th DAAAM International Symposium on Intelligent Manufacturing and Automation, DAAAM 2015 